\title{
Electroplating for high aspect ratio vias in PCB manufacturing: enhancement capabilities of acoustic streaming
}

\author{
Nadezhda Strusevich • Marc P. Y. Desmulliez • \\ Eitan Abraham • David Flynn · Thomas Jones • \\ Mayur Patel · Christopher Bailey
}

Received: 20 August 2013/ Accepted: 3 September 2013/Published online: 23 September 2013

(c) Shanghai University and Springer-Verlag Berlin Heidelberg 2013

\begin{abstract}
This paper considers the copper electrodeposition processes in microvias and investigates whether the quality of the electroplating process can be improved by acoustic streaming using megasonic transducers placed into a plating cell. The theoretical results show that acoustic streaming does not take place within the micro-via (either through or blind-via's), however it does help improve cupric ion transport in the area close to the mouth of a via. This replenishment of cupric ions at the mouth of micro-via leads to better quality filling of the micro-via through diffusion compared to basic conditions. Experiments showing the improved quality of the filling of vias are also presented.
\end{abstract}

Keywords Electronics manufacturing $\cdot$ Numerical modelling $\cdot$ High aspect ratio microvia .

Electroplating $\cdot$ Megasonic agitation $\cdot$ Acoustic streaming

\section{Introduction}

In modern microelectronic manufacturing, multi-layer (or 3D) packaging of the components placed on printed circuit boards (PCBs) is of crucial importance due to increasing requirements on performance and reliability of electronic devices and systems, as well as on their miniaturization.

N. Strusevich $(\bowtie) \cdot$ M. Patel · C. Bailey

School of Computing and Mathematical Sciences, Old Royal

Naval College, University of Greenwich , Park Row,

London SE10 9LS, UK

e-mail: N.Strusevich@gre.ac.uk

M. P. Y. Desmulliez · E. Abraham · D. Flynn · T. Jones School of Engineering \& Physical Sciences, Heriot-Watt University, Edinburgh EH14 4AS, UK
The connection between different layers of a package is provided by the so-called vertical interconnect access (via), which is a hole or cavity in the PCB filled with metal to facilitate the electrical conductivity of the interconnection. In this paper, we distinguish between blind vias exposed on one side of the board only, and through vias, open from both ends. Normally, the size of such a via is at the micrometer scale, and has a high aspect ratio (AR), i.e., the ratio of its height to its diameter. The most widely used technique for filling microvias is electrodeposition (ED) of metal (e.g., copper) into a microvia from an electrolyte solution.

Without going into technical details, a typical ED process used in microelectronics can be roughly described as follows. Panels, i.e., PCBs with predrilled or laser ablated microvias, are immersed into a plating cell filled with an electrolyte solution that contains ions of copper. In the presence of direct electric current, the metal ions are attracted to the panel and are deposited on the sides and the bottom of the microvias. This process is schematically shown in Fig. 1. The figure also zooms into a small part of the PCB showing a blind via.

A microvia can be seen as electroplated successfully if it is completely full, with no internal voids. However, under standard conditions, current crowding effects close to the mouths of the vias occur, so that the via closes prematurely, and copper ions are not replenished. This leads to ion depletion in the via and void formation (see Fig. 2).

Under basic ED conditions, it is assumed that the electrolyte is a still solution with no additives. As shown by numerical and real-life experiments [1], basic ED does not guarantee via fillings of an acceptable quality. This is usually attributed to insufficient ion transport into the via over time.

One of the approaches to enhancing the transport of cupric ions involves the use of inhibitors, accelerators and 


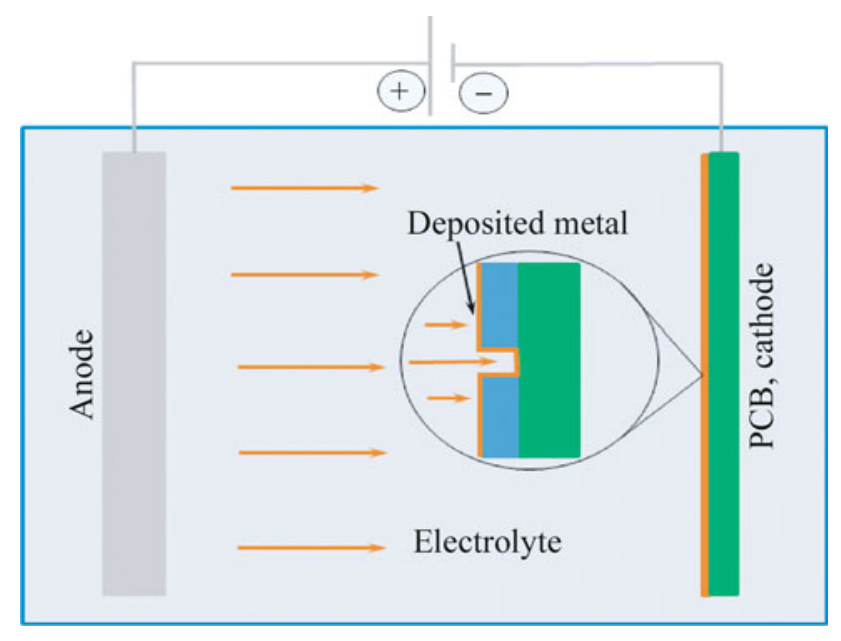

Fig. 1 Principal scheme of ED into a blind via in a plating cell

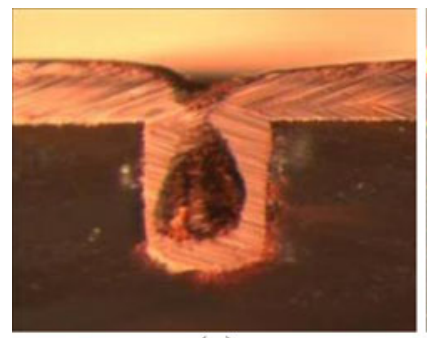

(a)

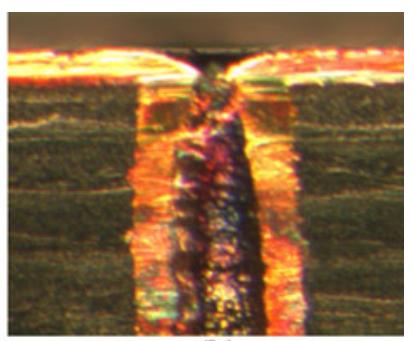

(b)
Fig. 2 Photos of (a) blind and (b) through vias with a closed mouth and voids inside

other chemical additives $[2,3]$. Another approach is related to applying a forced flow in the electrolyte. Such a flow can either be induced by certain mechanical means, such as pumping, or can be a result of megasonic agitation.

In the case of megasonic agitation, a plating cell is additionally equipped with a megasonic transducer. Propagation of high-power sound waves in liquid media is known to generate stream-like non-periodic movements, known as acoustic streaming (AS). According to Frampton et al. [4], AS is the creation of a steady fluid velocity from oscillating acoustic velocities.

We have investigated the use of numerical modelling techniques to verify whether AS, as one of the phenomena that accompanies megasonic agitation, can lead to an improved ion transport into the micro via, and eventually to an improved quality filling.

\section{Aspects of mathematical and numerical modeling of basic ED}

We consider the process of basic ED that satisfies the following assumptions: (i) at the cathode, only cupric ions are deposited; (ii) the anode does not change shape during
ED; (iii) the physical properties of the electrolyte remain constant; (iv) the activation overpotential $\eta$ at the cathode is constant; and ( $\mathrm{v}$ ) no use of additives or other forms of enhancement is assumed.

\subsection{Numerical modeling of basic ED}

Two processes are mainly responsible for ion transport during basic ED: diffusion which is caused by the difference in concentration of cupric ions, and migration which is caused by the electric field, i.e., positively charged cupric ions move towards the negatively charged cathode.

Let $C$ denote the concentration of the $\mathrm{Cu}^{2+}$ ions and $z=2$ be their charge. An equation that describes the rate of change of copper concentration in the electrolyte can be derived from the Nerst-Planck equation, and in our case this can be written as

$\frac{\partial C}{\partial t}=-D \nabla^{2} C-z \bar{\phi} \frac{F D}{R T} \nabla C+R_{C}$,

where $D$ the diffusivity of cupric ions in the electrolyte, $R$ the universal gas constant, $T$ temperature, and $F$ Faraday's number. The potential difference $\bar{\phi}$ is computed as the difference of potentials between the anode and the cathode divided by the distance $L$ between them. The value $\bar{\phi}$ can be taken as constant, since $L$ changes insignificantly during the ED process. Additionally, equation (1) includes a source term $R_{\mathrm{C}}$ that represents the amount of deposited moles of copper. Below, we refer to $R_{\mathrm{C}}$ as the sink. To compute it, we use Faraday's Law for the deposition rate $v$ given by

$v=\frac{\Omega i}{z F} \cdot \boldsymbol{n}$,

where $\Omega$ the atomic volume of copper, $\boldsymbol{n}$ the vector normal to the deposition surface, and $i$ the current density, i.e., the amperage of the ED current divided by the area of the deposition surface. We follow Refs. [6, 7] and throughout this paper apply the following formula for $i$, which is a version of the Butler-Volmer equation

$i=-i_{0} \frac{C^{\mathrm{int}}}{C^{\infty}} \exp \left(-\frac{\alpha z F \eta}{R T}\right)$

where $i_{0}$ the initial exchange current density, $C^{\text {int }}$ the molar concentration at the metal/electrolyte interface, i.e., in the vicinity of the cathode, $C^{\infty}$ the concentration in the far field, and $\alpha=1.5$ the dimensionless transfer coefficient.

Equation (1) is a form of the well-know general transport equation in computational fluid dynamics (CFD). One of the most popular approaches to solve these equations numerically is to solve their discrete analogues by the finite volume method (FVM). In particular, the FVM is implemented in the software multi-physics package PHYSICA, 
which is a modular suite of components for the simulation of coupled physical phenomena in 3D and time (see Ref. [8]).

We have developed and implemented in PHYSICA the explicit interface tracking method (EITM) for numerical modelling of basic ED in small features. The method allows the user to be permanently aware of the position of the interface between the electrolyte and the deposited metal. The way it is implemented makes the user be able to dynamically prescribe and change additional parameters, such as additional flow, acoustic streaming, etc., to various parts of the changing computational domain. The latter feature allows the EITM to be used as a modelling tool for various forms of enhanced ED.

The EITM under the conditions of basic ED has been validated against real-life experiments and known benchmarks [1]. The obtained numerical results demonstrate that basic ED does not guarantee a good quality filling of micro features, even for relatively low AR such as 1:1. The underperformance of basic ED should be seen as a strong motivation for the search of various methods of enhancement.

\subsection{Performance metrics of ED in microvias}

In this paper, we use via fill (VF) as a metric that allows us to assess the quality of filling.

$\mathrm{VF}$ is a quantitative measure of via filling that describes the effectiveness of the deposition process. It is a proportion of the filled part of the via and is defined in terms of the parameters shown in Fig. 3 as

$\mathrm{VF}=1-\left(d-h_{1}\right)^{2}\left(H-h_{2}\right) /\left(H d^{2}\right)$.

The values of VF closer to 1 correspond to a better quality of filling.

In our numerical experiments we are also interested in recording the filling time (FT), which is defined as the time elapsed either till complete filling of the via or until ion depletion. Smaller values of FT are preferable.

\section{Acoustic streaming phenomenon}

The study of AS in various media was initiated by Rayleigh in 1880s [10]. In our discussion of AS we mainly follow the classical work by Nyborg [11], who employs the method of successive approximations in order to derive the AS governing equations. According to this method the field variables (density, excess pressure, and velocity) are expressed as the sum of the first order small terms and the second order small terms. The first-order (acoustic) variables $\rho_{\mathrm{a}}, P_{\mathrm{a}}$ and $U_{\mathrm{a}}$ depend periodically on time with the angular frequency $\omega$ of the transducer. These variables

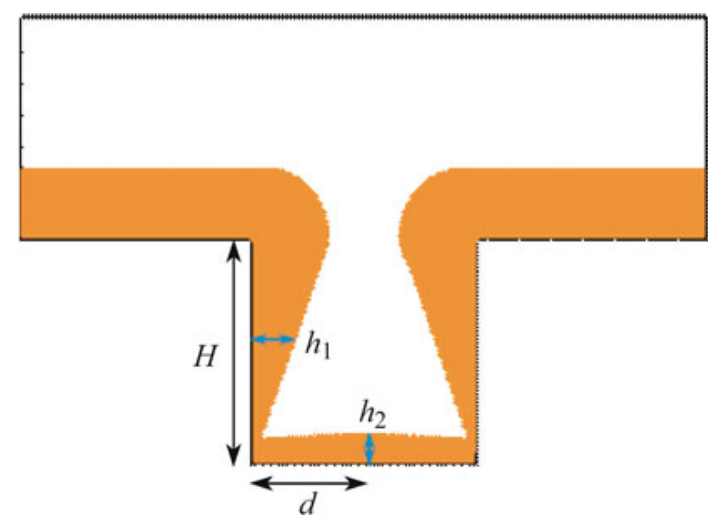

Fig. 3 Key dimensions in a trench

describe the oscillating group of variables and represent the sound field in the linear approximation. The second-order (streaming) variables $\rho_{\mathrm{s}}, P_{\mathrm{s}}$ and $U_{\mathrm{s}}$ include the time-independent terms.

In accordance with the method of successive approximations, the classical pair of equations for fluid medium, i.e., the equations of mass and momentum conservation, can be transformed to become

$\nabla P_{s}-\mu \nabla^{2} U_{s}=F$

where $\mu$ is dynamic viscosity. $F$ is the driving force given by

$-F=\left\langle U_{a} \nabla \cdot\left(\rho U_{a}\right)+\rho\left(U_{a} \nabla\right) \cdot U_{a} \cdot\right\rangle$

where \langle\rangle denotes time averaging.

For the purpose of this paper we are interested in several specific patterns of AS, which are associated with one of the following types of waves:

(i) a plane travelling wave that slips the walls of a wide channel;

(ii) a standing wave in a close-end narrow channel;

(iii) a travelling wave in an open-end narrow channel.

\section{Effect of AS on bulk ion concentration}

In our simulations of basic ED, the far field has to be chosen at a fairly large distance from the mouth of the via compared to the size of the via. The concentration $C^{\infty}$ in the far field is involved in equation (2) for the current density distribution.

As explained below, in the presence of megasonic agitation, the concentration $C^{\infty}$ can be prescribed to the region of the plating cell that is immediately close to the mouth of the via.

Assume that a megasonic transducer is placed in a plating cell, so that its oscillating surface is perpendicular 
to the surfaces of the panels (PCBs). Here we deal with waves of type (i) above. Along each side of a panel, the acoustic boundary layer is formed, which is known to be extremely thin. For example, as follows from Ref. [11], its thickness $\delta$ is computed by

$\delta=\sqrt{\frac{2 \mu}{\omega \rho}}$

and for a transducer's frequency of $1 \mathrm{MHz}$ is less than $0.6 \mu \mathrm{m}$. The velocity $U_{s}$ in the acoustic boundary layer is high and responsible for fast replenishment of ions along the panel. Thus, without loss of accuracy, in micro models that study ion transport in the presence of megasonic agitation, we may prescribe the bulk concentration $C^{\infty}$ to the area immediately close to the mouth of a microvia.

It is worth mentioning that such a change cannot be done in the presence of an ordinary flow and generated by mechanical means. It should be recalled that an ordinary flow generates the hydrodynamic boundary layer along the panel. As estimated in Ref. [12], with a flow velocity of $4 \mathrm{~m} / \mathrm{s}$, the thickness of this layer reaches $1,500 \mu \mathrm{m}$. Thus, in the case of an induced ordinary flow we cannot assign the bulk concentration at smaller distances from the surface of the panel.

We have conducted a series of numerical experiments, based on the EITM, to compare the outcomes of ED under basic conditions (basic ED model) and ED with the modified bulk concentration boundary conditions (partial ED model). The corresponding computational domains for a trench are shown in Fig. 4 (the left half of geometry). Notice that we use the term "partial" because the model does not include the streaming phenomena inside the via.

In this section, we report on the numerical results for blind vias only, and a similar study on through vias is presented in Sec. 5.2.

In our simulations, the following input parameters have been used: temperature $25^{\circ} \mathrm{C}$, diffusivity $2 \times 10^{-9} \mathrm{~m}^{2} / \mathrm{s}$, anode potential $0.135 \mathrm{~V}$, cathode potential, $-0.135 \mathrm{~V}$, overpotential $-0.09 \mathrm{~V}$, initial current density $50 \mathrm{~A} / \mathrm{m}^{2}$. We have conducted numerical experiments with two trenches, both of width $w$ is equal to $4 \mu \mathrm{m}$. One trench of height $h$ is equal to $4 \mu \mathrm{m}(1: 1 \mathrm{AR})$, while for the other one $h$ is equal to $8 \mu \mathrm{m}(2: 1 \mathrm{AR})$. For a 1:1 AR trench, the bulk concentration is chosen to be $100 \mathrm{~g} / \mathrm{L}$, while for the $2: 1 \mathrm{AR}$ trench the concentration is $200 \mathrm{~g} / \mathrm{L}$.

The numerical results in terms of the applied metrics VF and FT are given in Table 1.

For each trench, the filling times for both models are similar. Figure 5 shows the differences between the deposition level achieved in each trench for the basic and the partial models (the left half of geometry). The main difference is that under the conditions of the basic model none of the vias is completely filled. By contrast, in the case of

\section{Bulk Concentration}

(a)

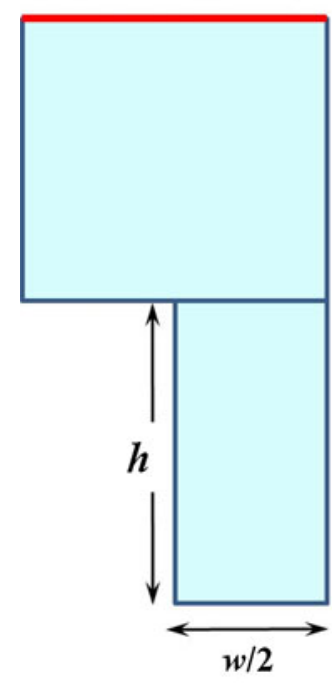

(b)

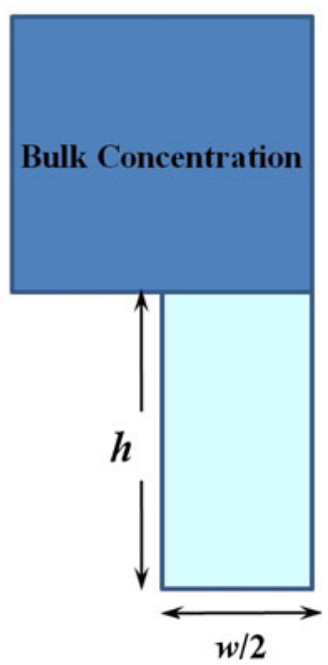

Fig. 4 Computational domain for (a) Basic ED model; (b) Partial ED model

Table 1 Numerical results for Basic and Partial ED models for blind vias

\begin{tabular}{llllll}
\hline Model & $1: 1 \mathrm{AR}$ & & & $2: 1 \mathrm{AR}$ & \\
\cline { 2 - 3 } \cline { 6 - 6 } & FT $(\mathrm{s})$ & $\mathrm{VF}$ & & FT $(\mathrm{s})$ & $\mathrm{VF}$ \\
\hline Basic & 31.0 & 0.9993 & & 30.0 & 0.9950 \\
Partial & 30.0 & 1.0000 & & 31.0 & 1.0000 \\
\hline
\end{tabular}

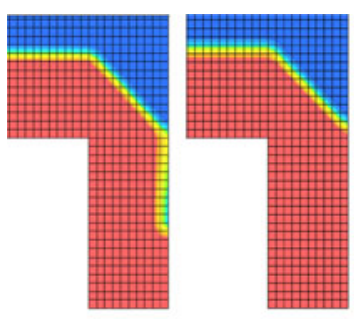

(a)

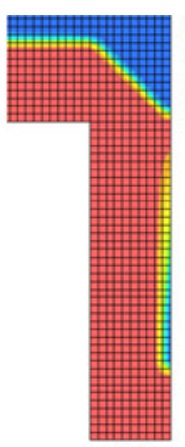

(c)

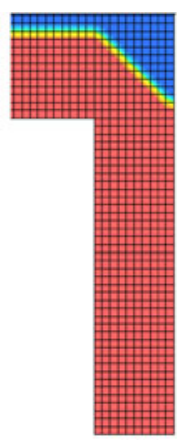

(d)
Fig. 5 Deposition level for the 1:1 AR via: (a) Basic model; (b) Partial model, and for 2:1 AR via: (c) Basic model; (d) Partial model

the partial model the filling of both vias is complete, leaving no void.

Thus for the blind vias, the presence of AS has an essential effect on ion transport and eventually on the quality of filling. This is due to a permanent supply of copper ions at the mouth area, which is created by AS along the panel. 


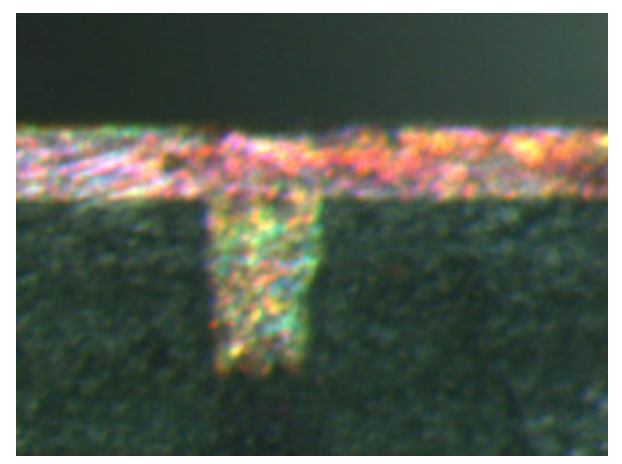

Fig. 6 Blind via with 1.7:1 aspect ratio filled with void free $\mathrm{Cu}$

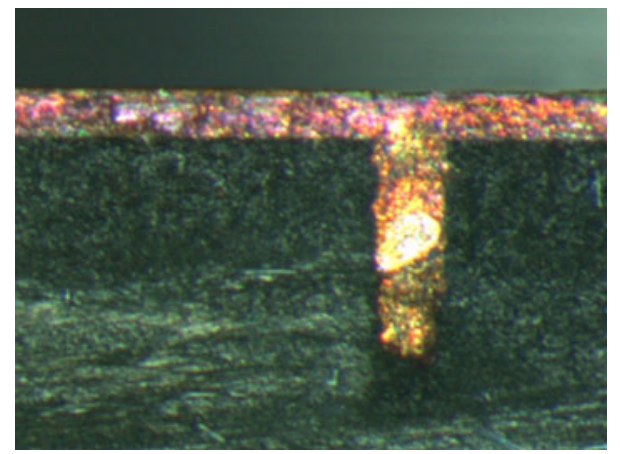

Fig. 7 Blind via with a 3:1 aspect ratio filled with void free $\mathrm{Cu}$ (No seed layer is present at the bottom of the via)

These simulations are being supported with experiments carried out on a large scale via of diameter of $200 \mu \mathrm{m}$. For example, DC deposition at low current density of $1 \mathrm{~A} / \mathrm{dm}^{2}$ with $100 \mathrm{~W}$ megasonic power leads to void free filling of a blind via of 1.7:1 AR as shown in Fig. 6. The VF is 99\%

The maximum blind via aspect ratio, limited due to seed layer deposition within the vias, was equal to 3:1. Figure 7 shows that DC deposition with megasonic agitation can fill such a via with void free $\mathrm{Cu}$.

\section{Acoustic streaming inside the microvia}

By conducting numerical experiments with a plating cell equipped by a megasonic transducer, we are able to determine a distribution of the amplitude $A$ of the acoustic velocity $U_{\mathrm{a}}$ on the surfaces of the panels. This can be achieved by standard software, such as COMSOL multiphysics. Then, with the value of $U_{\mathrm{a}}$ taken at the mouth of the via we can use Eqs. (4) and (5) to determine the streaming velocity $U_{\mathrm{s}}$ inside the via.

\subsection{Acoustic streaming in blind vias}

If the via is blind, we deal with a wave of type (ii) introduced in Sec. 3. Such a wave travels inside the feature until it hits its bottom, which is essentially a cathode. In the case of ED of copper, the trench has a highly reflective bottom. It can be shown that more than $90 \%$ of energy of the incident wave will be reflected and a standing wave will be formed. Thus the ions will not be carried by that wave, and no additional improvement of ion transport will be observed. This conclusion is supported by many authors in Ref. [13].

\subsection{Acoustic streaming in through vias}

The situation is different for a through via, since now we deal with a wave of type (iii) introduced in Sec. 3. In this case, we set up a full model of ED, which further extends the partial model by incorporating the streaming along the walls of the via.

For an open end via, let $x$ be the distance from a vertical via wall. To model the streaming velocity $U_{s}$ in the downward $y$-direction inside the via, we use equation (5) and the results in Ref. [4, 11], where an analytical expression for the driving force $F$ for a wave of type (ii) is written as the sum of two parts: $F^{(v)}$ that represents the body force due to viscous losses (away from the boundary layer, for $x>\delta$ ), and $F^{(b)}$ which is due to an acoustic wave grazing a rigid boundary (in the boundary layer, i.e., for $0<x<\delta$ ). The full representation of $F$ is given below:

$$
\begin{aligned}
& F=F^{(v)}+F^{(b)}, \\
& F^{(v)}=A^{2} \alpha \rho \exp (2 \alpha y), \\
& F^{(b)}=\frac{1}{2} A^{2} \rho \exp (2 \alpha y)\left(k\left(C+S-\exp \left(-\frac{2 x}{\delta}\right)\right)\right. \\
& \left.\quad+\alpha\left(\exp \left(-\frac{2 x}{\delta}\right)-3 C+S\right)\right),
\end{aligned}
$$

where

$C=\exp (-x / \delta) \cos (x / \delta), S=\exp (-x / \delta) \sin (x / \delta)$.

Of the two components that form $F$, the weaker one is $F^{(v)}$, attributed to viscous effects, and a larger force $F^{(b)}$ that acts in the vicinity of an acoustic boundary layer along the walls. We use $F$ for computing the streaming velocity $U_{\mathrm{s}}$.

Numerical experiments have been conducted, based on the EITM, to compare the outcomes of ED in through vias for the basic, partial and full ED models. Both vias are of 2:1 AR, of width $4 \mu \mathrm{m}$ and $100 \mu \mathrm{m}$, and the bulk concentration is chosen to be $200 \mathrm{~g} / \mathrm{L}$ for both vias. The other input parameters are the same as in our simulations for trenches, stated in Sec. 4, except the initial current density is $150 \mathrm{~A} / \mathrm{m}^{2}$ and the overpotential is $-0.09 \mathrm{~V}$. The acoustic parameters are the transducer intensity of agitation $I$ is equal to $5 \mathrm{~W} / \mathrm{cm}^{2}$, frequency $f$ is equal to $1 \mathrm{MHz}$, attenuation coefficient $\alpha$ is equal to $0.0078 \mathrm{~m}^{-1}$. 
Table 2 Numerical results for three ED models in through vias

\begin{tabular}{llllll}
\hline Model & \multirow{2}{*}{$w=4 \mu \mathrm{m}$} & & \multicolumn{2}{c}{$w=100 \mu \mathrm{m}$} \\
\cline { 2 - 3 } \cline { 6 - 7 } & FT (s) & VF & & FT (s) & VF \\
\hline Basic & 11.8 & 0.749 & & 310.0 & 0.367 \\
Partial & 11.8 & 0.976 & 310.0 & 0.707 \\
Full & 11.8 & 0.977 & & 301.0 & 0.721 \\
\hline
\end{tabular}

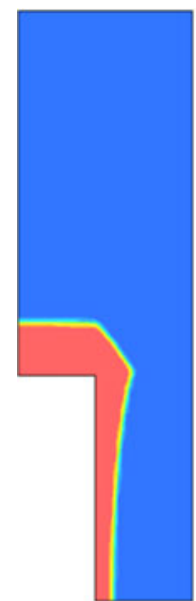

(a)

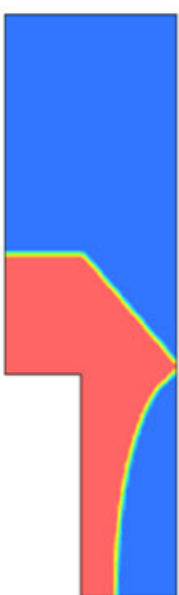

(b)

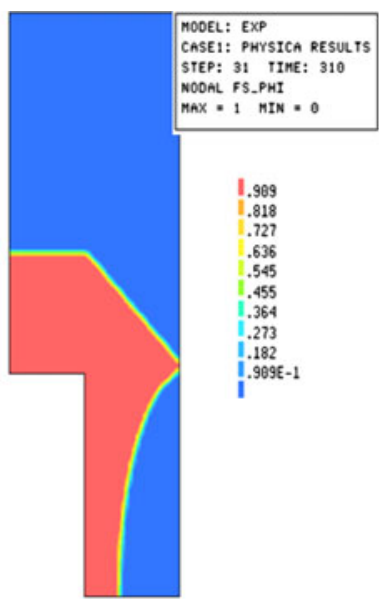

(c)
Fig. 8 Deposition level for 2:1 AR through via for (a) basic, (b) partial and (c) full ED models

The numerical results in terms of the applied metrics VF and FT are given in Table 2.

The following observations can be made. For all three models, the filling time is the same, i.e., $11.8 \mathrm{~s}$ for the $8 \mu \mathrm{m} \times 4 \mu \mathrm{m}$ via and $310 \mathrm{~s}$ for the $200 \mu \mathrm{m} \times 100 \mu \mathrm{m}$ via. In all cases, the ED process stops due to depletion and a void is formed. For the $8 \mu \mathrm{m} \times 4 \mu \mathrm{m}$ via, the results for the partial and full models are virtually indistinguishable. For the $200 \mu \mathrm{m} \times 100 \mu \mathrm{m}$ via, the full model provides a slightly better value of VF than that achieved in the Partial model.

For the $200 \mu \mathrm{m} \times 100 \mu \mathrm{m}$ through via, Fig. 8 gives a visual representation of the obtained results after $310 \mathrm{~s}$ of deposition (the top-left quarter of geometry). For the partial and full ED models, a high concentration is maintained in the area above the via, but as the mouth of the via closes, the simulation stops due to a low concentration in the void area. For the basic ED model, the via is far from being filled during the same $310 \mathrm{~s}$. On the other hand, due to a faster deposition rate in the case of the partial and full ED models, a considerable crowding effect is observed in the corner regions.

Thus, the AS inside through has only a minor impact on the ion transport.

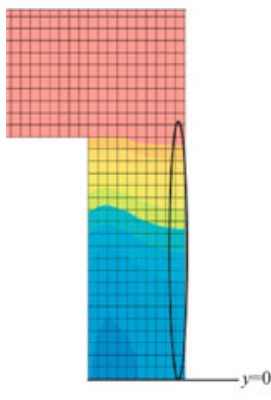

(a)

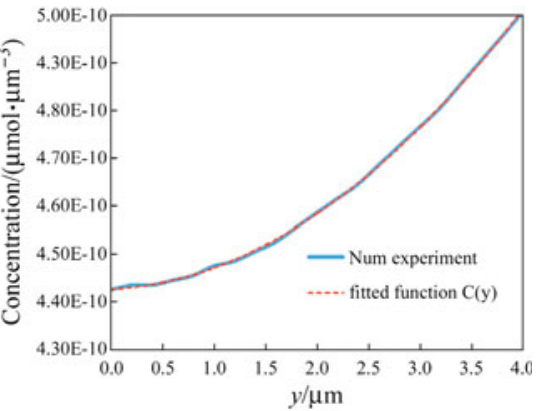

(b)
Fig. $98 \mu \mathrm{m} \times 4 \mu \mathrm{m}$ via: (a) concentration distribution with the cells along the vertical centre encircled; (b) the data with the fitted function

\subsection{Impact of acoustic streaming in a through via}

As demonstrated in Sec. 5.2, there is a small difference in performance of the partial and full ED models. In this section, we provide other evidence that the impact of acoustic streaming inside a microvia is insignificant, by estimating and comparing the diffusion and the convective terms in the equation for concentration in the form

$\frac{\partial C}{\partial t}=-D \nabla^{2} C-U_{\mathrm{s}} \nabla C+R_{\mathrm{C}}$

We have conducted computational simulations for 2:1AR through vias with width $4 \mu \mathrm{m}$ and $100 \mu \mathrm{m}$, the same as in the numerical experiments discussed in Sec. 5.2. For each via, we fix a certain time $t_{0}$ since the beginning of simulation and then take records of the concentration values along the central vertical cross section of the via. For the obtained array of data, we use regression to find the polynomial function $C(y)$ that approximates the concentration distribution (in $\mu \mathrm{mol} / \mu \mathrm{m}^{3}$ ) along this cross section, where $y$ is the distance from the geometric centre of the via.

We have taken $U_{s}=6 \mu \mathrm{m} / \mathrm{s}$, which corresponds to the AS velocity in the middle part of a via for the given input amplitude $A$. For the $8 \mu \mathrm{m} \times 4 \mu \mathrm{m}$ via, our approach is illustrated in Fig. 9.

For the $8 \mu \mathrm{m} \times 4 \mu \mathrm{m}$ via and $t_{0}=4 \mathrm{~s}$, regression gives $C(y)=3 \times 10^{-12} y^{2}+10^{-12} y+4 \times 10^{-10}$ with $R^{2}=0.9998$. For the $200 \mu \mathrm{m} \times 100 \mu \mathrm{m}$ via and $t_{0}=60 \mathrm{~s}$, regression gives $C(y)=6 \times 10^{-14} y^{2}+10^{-13} y+7 \times 10^{-10}$ with $R^{2}=0.9999$.

The diffusion and convective terms are computed by finding the relevant derivatives of the functions $C(y)$ for each via; the diffusion terms have being computed for $y=0$. The results of the corresponding computation are shown in Table 3.

It follows that the contribution of the convective term is much smaller than that of the diffusion term: 2,000 times for the $8 \mu \mathrm{m} \times 4 \mu \mathrm{m}$ via and 400 times for the 
Table 3 Comparison of the convective and the diffusion terms

\begin{tabular}{lll}
\hline Size of the via & Diffusion term & Convective term \\
\hline $8 \mu \mathrm{m} \times 4 \mu \mathrm{m}$ & $1.2 \times 10^{-8}$ & $6 \times 10^{-12}$ \\
$200 \mu \mathrm{m} \times 100 \mu \mathrm{m}$ & $2.4 \times 10^{-10}$ & $6 \times 10^{-13}$ \\
\hline
\end{tabular}

$200 \mu \mathrm{m} \times 100 \mu \mathrm{m}$. Since the convective term reflects the influence of the AS inside the via, the fact that it is strongly dominated by the diffusion term explains that the impact of AS inside a through vias on ion transport is negligible.

\section{Conclusions}

Acoustic streaming makes a considerable difference on ion transport in microvias by replenishing cupric ions in the vicinity of the mouth of the via. This allows us to introduce a modified boundary condition that assumes the same bulk concentration in the whole area above the mouth of the feature, rather than at the far field. For the resulting partial ED models a higher deposition rate is observed for both blind and through vias. The same features under the conditions of the partial model appear to be more filled than under the conditions of the basic ED models; for smaller trenches a complete filling has been achieved. Overall quality of filling for the partial models is still dubious, with a considerable crowding effect at the mouth.

For trenches, AS inside the via has no major influence, which follows from both theoretical considerations regarding AS in narrow closed channels with a reflective bottom and real-life experiments, e.g., Ref. [14].

For through vias, the presence of AS inside the via makes no essential influence on ion transport.

It remains a goal for further research to study the influence of other phenomena that accompany megasonic agitation on ion transport as it is evident from experiments that enhanced filling of the via is witnessed. This might include possible heat effects and formation of resonant bubbles.
Acknowledgements The authors would like to acknowledge the Engineering and Physical Sciences Research Council (EPSRC) for their financial support through the grant ASPECT supported by the Scottish Manufacturing Institute (SMI) at Heriot-Watt University. We also acknowledge very productive discussions with Dennis Price from Merlin Circuit technology Ltd who provided a valuable insight into the electroplating process at industrial scale.

\section{References}

1. Strusevich N, Bailey C, Costello S, Patel M, Desmulliez MPY (2013) Numerical modeling of electroplating process for microvia fabrication. In: Euro SimE conference, Wroclaw, Poland, April 2013

2. Andricacos PC, Uzoh C, Dukovic JO, Horcans J, Deligianni H (1998) Damascene copper electroplating for chip interconnects. IBM J Res Dev 42(5):567-574

3. Moffat TP, Wheeler D, Kim SK, Josell D (2006) Curvature enhanced adsorbate coverage model for electrodeposition. J Electrochem Soc 153(2):C127-C132

4. Frampton KD, Martin SE, Minor K (2003) The scaling of acoustic streaming for application in micro-fluidic devices. Appl Acoust 64:681-692

5. Kirby BJ (2010) Micro- and nanoscale fluid mechanics: transport in microfluidic devices. Cambridge University Press, Cambridge

6. Low CTJ, Roberts EPL, Walsh FC (2007) Numerical simulation of the current, potential and concentration distributions along the cathode of a rotating cylinder hull cell. Electrochim Acta 52:3831-3840

7. Wheeler D, Josell D, Moffat TP (2003) Modeling superconformal electrodeposition using the level set method. J Electrochem Soc 150(5):C302-C310

8. PHYSICA, Multiphysics Software Ltd., London, 2000. http:// www.multi-physics.com

9. Fang C (2011) Croissance electrolytique du cuivre appliquii a la technologie system in package. Dissertation, University of Rennes

10. Rayleigh L (1945) The theory of sound. Dover, New York

11. Nyborg WLM (1965) Acoustic streaming. In: Physical acoustics, principles and methods, vol 2B. Academic Press, New York, pp 265-331

12. Gale GW, Busnaina AA (1999) Roles of cavitation and acoustic streaming in megasonic cleaning. Part Sci Technol 17:229-238

13. Nilson RH, Griffiths SK (2002) Enhanced transport by acoustic streaming in deep trench-like cavities. J Electrochem Soc 149(4):G286-G296

14. Liu G, Huang X, Xiong Y, Tian Y (2008) Fabrication HARMS by using megasonic assisted electroforming. Microsyst Technol $14: 122-1223$ 